\title{
Capillaroscopy in Fabry disease: Study of a family
}

\author{
Mónica López-Rodríguez, Francisco Javier Barbado-Hernández, Antonio Torrijos Eslava', \\ Julia García-Consuegra², Natividad Arias-Martínez, Jorge Gómez-Cerezo, Bárbara Pagán-Muñoz, \\ Juan José Vázquez-Rodríguez \\ Department of Internal Medicine, University Hospital La Paz, Madrid, Spain, 'Department of Rheumatology, University Hospital La Paz, \\ Madrid, Spain, ${ }^{2}$ Department of Chidren Rheumatology, University Hospital La Paz, Madrid, Spain
}

BACKGROUND: Nail bed capillaroscopy represents a simple and innocuous diagnosis method, used in vasculitis or collagen diseases. In Fabry disease (FD), there is a vasculopathy due to the storage of globotriaosylceramide. This is the first series that studies capillaroscopy in FD. OBJECTIVE: To describe and evaluate the capillary findings in Fabry patients.

MATERIALS AND METHODS: Eight Fabry patients were selected; five were under enzyme replacement therapy (ERT) with agalsidase-A.

RESULTS: In three patients (under ERT), the capillaroscopy showed no abnormalities; two patients had absence of some capillaries (one with ERT); in three (one with ERT) the capillaroscopy revealed ramification of capillaries. $\mathrm{Pa}$ tients with normal capillaroscopy had no symptom of acroparesthesia for more than a year.

CONCLUSIONS: Capillaroscopy is a good method to evaluate small vessels in FD and seems to have a correlation with ERT benefits, clinical manifestations and capillary findings. We purpose to use the capillaroscopy in the study of Fabry patients.

Key words: Capillaroscopy, fabry disease, acroparesthesia, enzyme replacement therapy

\section{Introduction}

Fabry disease (FD) is a metabolic, rare and severe disease, due to the storage of a glycosphingolipid, the globotriaosylceramide (Gb3), in lysosomes from vascular endothelium and lysosomes from organs like kidney, heart and nervous system. It is an inborn disorder caused by an inherited defect of the a-galactosidase $A$ gene. This gene is located on the X-chromosome (Xq22.1). Therefore, the disease has an X-linked inheritance pattern. According to the Lyon hypothesis, the random inactivation of the $\mathrm{X}$-chromosome is a primordial factor for the development of signs and symptoms in heterozygous females.

The prevalence of FD is $1 / 117.000$, but this number may be higher, because there are numerous cases in which correct diagnosis is obtained very late due to the difficulty that offers this entity (sometimes considered as a "great simulator"). ${ }^{[1]}$ The main clinic manifestations are neuropathic pain, acroparesthesia, angiokeratomas and, subsequently, renal failure (first cause of death in these patients), ${ }^{[2]}$ cardiomyopathy and nervous system affectation. Nowadays, thank to enzyme replacement therapy (ERT), perspectives about prognosis and quality of life are quite auspicious and very different to those we had few years ago. ${ }^{[3]}$

In FD, there is a vasculopathy due to the storage of Gb3 and, also, immunocomplex. Endothelial cells and smooth muscular cells of the vascular wall have cumulus of this glycosphingolipid inside them. This progressive deposit, may cause ischemia and infarct (main manifestations of FD) and multisystemic affection. In fact, it has been considered as a "pseudovasculitis" of small vessels..$^{[4,5]}$ This feature may be implicated in the physiology of some signs and symptoms, for example, acroparesthesia.

Nail bed capillaroscopy represents a simple and innocuous diagnosis method. It has been used in a large number of disorders, mainly vasculitis and collagen diseases. The capillary examination has not been described previously, excepting two anecdotic cases. ${ }^{[6,7]}$ This is the first series that studies the nail bed capillaroscopy in FD. 
The present study was undertaken to determine and evaluate the capillary findings in Fabry patients and also to analyze the relationship with the clinic manifestations, especially acroparesthesia and the effect of ERT in these patients.

\section{Materials and Methods}

Eight members of the same family, all of them with FD, were selected (5 females, 3 males); five of them were under enzyme replacement therapy (ERT) with agalsidase A. None of the eight patients was smoker. Pedigree of this family is described in Figure 1.

The nail bed capillaroscopy was performed with a Nikon SMZ-10 stereomicroscopy, according to usual techniques. In patients with ERT, capillaroscopy was performed the twelfth day after the infusion of agalsidase A (they receive the enzyme every fifteen days). The rheumatologist who made the capillary test did not know who of the eight patients were under ERT, or not.

Capillaroscopy is considered as normal when the capillaries show loop morphology, (without ramifications) and their position is parallel to the cutaneous surface and to the adjoining ones; the nail bed capillary density consists of approximately 10 capillaries $/ \mathrm{mm}^{8}$.

\section{Results}

In three patients, (all of them under ERT), the capillaroscopy did not show significant abnormalities. Two patients had absence of some capillaries, with less

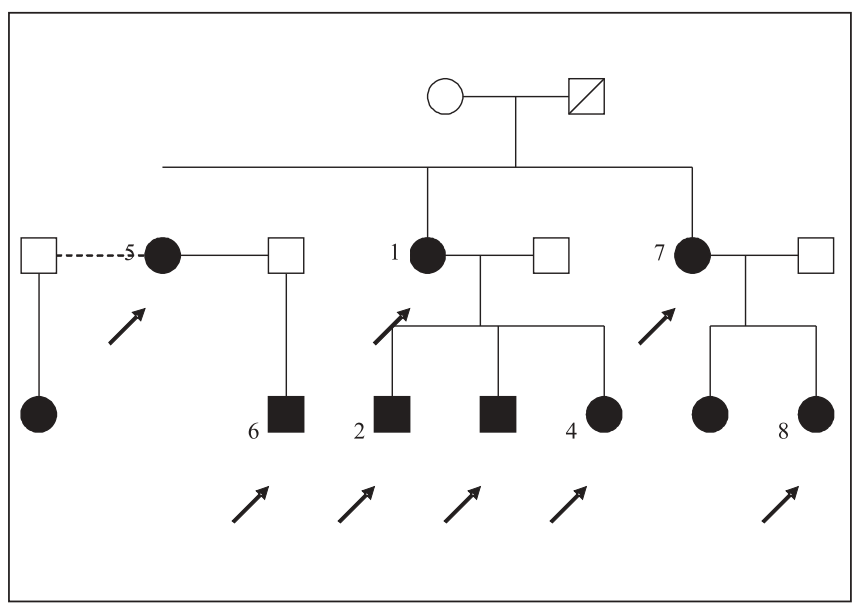

Figure 1: Pedigree of the family. Arrows indicate the patients who were practiced capillaroscopy than 5 capillaries $/ \mathrm{mm}$ (one with ERT). And finally, in three more patients (only one of them with ERT) the capillaroscopy revealed fasciculated ramifications of some capillaries, in 3-4 loops [Figures 2, 3].

In the group of patients with normal capillaroscopy, there was no symptom of acroparesthesia for more than a year ago.

Clinic characteristics of the patients and capillaroscopy findings are summarized in Table 1.

\section{Discussion}

FD is a multisystemic disorder with severe manifestations, like stroke and cardiac attack. These complications and the renal involvement, can lead to the early death of these patients. The damage is caused by the storage of Gb3 in cells from heart and central nervous system and, also in endothelial cells of their

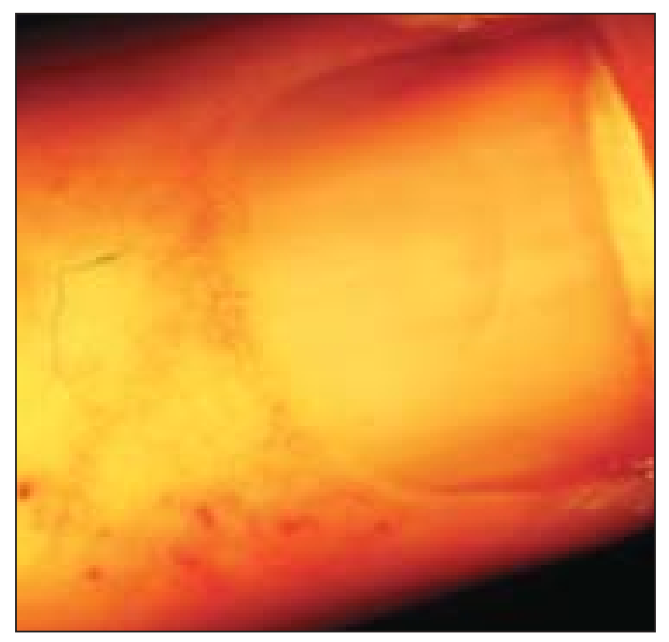

Figure 2: Patient 8. Nail bed capillaroscopy in a fabry patient with some ramifications

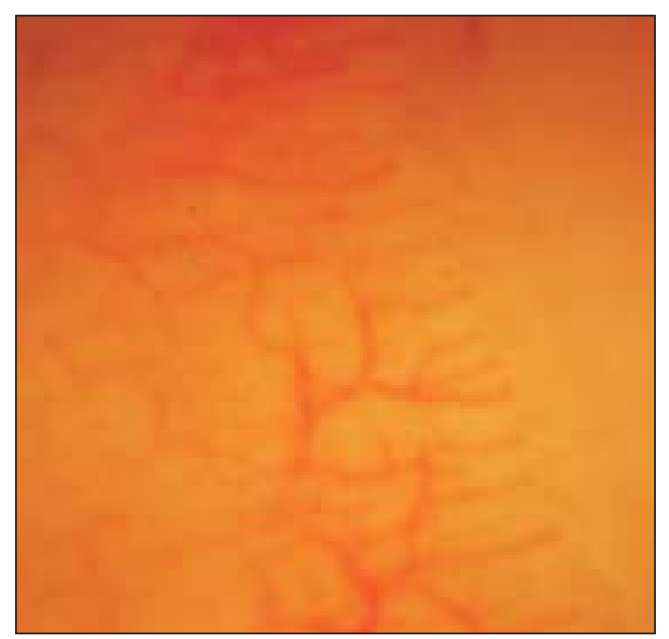

Figure 3: Patient 4. Fasciculated ramifications of some capillaries 
Table 1: Clinical characteristics and capillaroscopy findings

\begin{tabular}{|c|c|c|c|c|c|c|}
\hline Age & Gender & Acroparesthesia & Other manifestations & ERT & Time of ERT & Capillary finding \\
\hline 53 & Female & Yes (sometimes) & Joint pain. Proteinuria & Yes & 15 months & Absence some capillaries \\
\hline 30 & Male & No & Proteinuria facial dismorphia & Yes & 38 months & No abnormalities \\
\hline 26 & & & Proteinuria Facial dismorphia & Yes & 38 months & No abnormalities \\
\hline 20 & Female & Yes (sometimes) & & Yes & 38 months & $\begin{array}{l}\text { Fasciculated ramifications of } \\
\text { some capillaries }\end{array}$ \\
\hline 15 & Male & Yes (sometimes) & & No & & $\begin{array}{c}\text { Fasciculated ramifications } \\
\text { of some capillaries } \\
\text { Post-traumatic } \\
\text { microhemorrhages }\end{array}$ \\
\hline 50 & Female & Yes (frequently) & Chronic pain & No & & Absence some capillaries \\
\hline
\end{tabular}

cerebral and coronary arteries.

Capillaroscopy is a very good method to evaluate small vessels in FD with no risk for the patient. This technique has a correlation between some clinical manifestations and the capillary findings: the three patients with normal capillaroscopy did not have symptoms of acroparesthesia. And, what is much more important, these three patients were under treatment with agalsidase A. In fact, ERT seems to result effective stopping and, even, improving the storage of Gb3 in small vessels.

In the both two anecdotic FD cases with nail bed study, the capillaroscopy findings were similar to ours, principally fasciculated ramifications of some capillaries.

A study from our service analyzed the utility of capillaroscopy in vasculitis. ${ }^{[9]}$ In this article some similarities were found with the present study. For example, absence of capillaries or micro-hemorrhages.

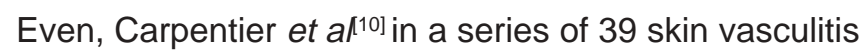
found high prevalence of capillary ramifications in their samples, translating vascular neoformation.

Thus, if we have an easy and non-invasive technique to see how these vessels are, we will be able to predict the potential risk of these patients to suffer some of these complications. And, probably we will have a good tool to asses the response of every patient to the ERT.

Since this is a very simple, available and innocuous method, we purpose to use the capillaroscopy in the regular study of Fabry patients. And even, as a marker of response to treatment, which would have a great value.

\section{References}

1. Nakao S, Takenaka T, Maeda M, Kodama C, Tanaka A, Tahara M, et al. An atypical variant of Fabry's disease in men with left ventricular hypertrophy. $\mathrm{N}$ Engl J Med 1995;333:288-93.

2. Thadhani R, Wolf M, West ML, Tonelli M, Ruthazer R, Pastores GM, et al. Patients with Fabry disease on dialysis in the United States. Kidney Int 2002;61:249-55.

3. Schiffmann R, Kopp JB, Austin HA 3rd, Sabnis S, Moore DF, Weibel T, et al. Enzyme replacement therapy in Fabry disease. JAMA 2001;285:2743-9.

4. Sack KE. When vasculitis is not vasculitis. Hospital Practice 1993;28:94-103.

5. Lie JT. Vasculitis, 1815 to 1991: classification and diagnostic specificity. J Rheumatol 1992;19:83-9.

6. Jansen W, Lentner A, Genzel I. Capillary changes in angiokeratoma corporis difusum Fabry. J Dermatol Sci 1994;7:68-70.

7. Frank J, Jansen-Genzel W, Lentner A, Wienert V. Fabry's disease (Angiokeratoma corporis diffusum). Hautarzt 1996;47:776-9.

8. Franco A, Carpentier P. Nail bed capillaroscopy. Abbott Laboratories 1981.

9. Sendino Revuelta A, Barbado Hernandez FJ, Torrijos Eslava A, Gonzalez AI, Pena Sanchez de Rivera JM, Vazquez Rodriguez JJ. Capillaroscopy in vasculitis. An Med Int 1991;8:217-20.

10. Drevet JG, Carpentier PH, Lelong C. Nail bed capillaroscopy in rheumatoid arthritis. Prospective study of 80 cases. Rev Rheum 1986;53:361-71. 